\title{
The KM3NeT project: status and perspectives
}

\author{
A. Margiotta ${ }^{1, *}$ \\ ${ }^{1}$ Dipartimento di Fisica dell'Università and Sezione INFN - Bologna, viale C. Berti Pichat, 6/2, 40127 Bologna, Italy \\ * on behalf of the KM3NeT Consortium
}

Correspondence to: A. Margiotta (margiotta@bo.infn.it)

Received: 28 July 2012 - Published in Geosci. Instrum. Method. Data Syst. Discuss.: 16 August 2012

Revised: 14 December 2012 - Accepted: 17 December 2012 - Published: 23 January 2013

\begin{abstract}
KM3NeT is an international consortium involving more than 300 scientists from $10 \mathrm{EU}$ countries. Its main objective is the construction of a multi- $\mathrm{km}^{3}$ high-energy neutrino telescope in the Mediterranean Sea that will also host an interdisciplinary observatory for marine sciences. $\mathrm{KM} 3 \mathrm{NeT}$ has been included in the roadmap of the European Strategy Forum of Research Infrastructures (ESFRI). Very high energy neutrinos are important messengers to study non-thermal phenomena in the Universe. The pioneering ANTARES, NEMO and NESTOR underwater neutrino telescope projects include the extensive R\&D knowledge base behind the KM3NeT project. A Technical Design Report has been published that describes the technological solutions chosen for the detector. The present status of the project is presented.
\end{abstract}

\section{Introduction}

Neutrinos are the perfect probe to explore the far Universe. They have no electrical charge, are insensitive to gravitational and magnetic fields and interact only weakly. This means they can travel huge distances from their production sites before reaching a detector on the Earth, transporting direct information on mechanisms acting inside cosmic accelerators. The discovery of astrophysical neutrinos will open a new perspective of astronomy and astrophysics, complementing present gamma ray astronomy and cosmic ray studies.

The same properties that make them unique messengers of high energy processes represent severe limitations to their detectability. Very large detection volumes, at least $1 \mathrm{~km}^{3}$, are required in order to have an unambiguous signal due to astrophysical neutrinos together with an effective shield against the overwhelming background due to atmospheric muons, residuals of the high energy cosmic ray showers. $\mathrm{A} \mathrm{km}^{3}$ size neutrino telescope is currently in data taking at the South Pole, IceCube (Abbasi et al., 2012). However, its sensitivity to sources in the southern sky, which includes most of the Galactic plane and the Galactic center, is greatly reduced. These regions host several possible sources of high energy neutrinos (Fig. 1).

In order to cover the full sky and to observe the Galactic plane, a multi- $\mathrm{km}^{3}$ sized detector in the Mediterranean Sea is needed (Fig. 2; Abbasi et al., 2011).

$\mathrm{KM} 3 \mathrm{NeT}$ is a deep sea multidisciplinary observatory in the Mediterranean Sea that will host a $\mathrm{km}^{3}$ size neutrino telescope and Earth and sea science devices dedicated to the continuous monitoring of the deep sea environment. In the framework of the EU FP6, a KM3NeT Design Study (DS) project was funded in 2006. In 2008, a Preparatory Phase (PP) project was funded by the EU through FP7. Both projects played a fundamental role to arrive at a reliable technical design and allowed addressing the financial, governance and strategic issues to be faced before undertaking the construction. Clarifying the scientific potential of KM3NeT led to its inclusion in the priority list of ESFRI (European Strategy Forum on Research Infrastructures) and to strong supporting recommendations in the European Roadmaps of Astroparticle Physics and of Astronomy. Recently, significant funds have been made available for infrastructure construction at two sites of the Mediterranean Sea: in front of the southern French coast and in proximity to Capo Passero, Sicily (Italy). They will not allow the completion of the whole detector, but represent a first important step towards this goal. 


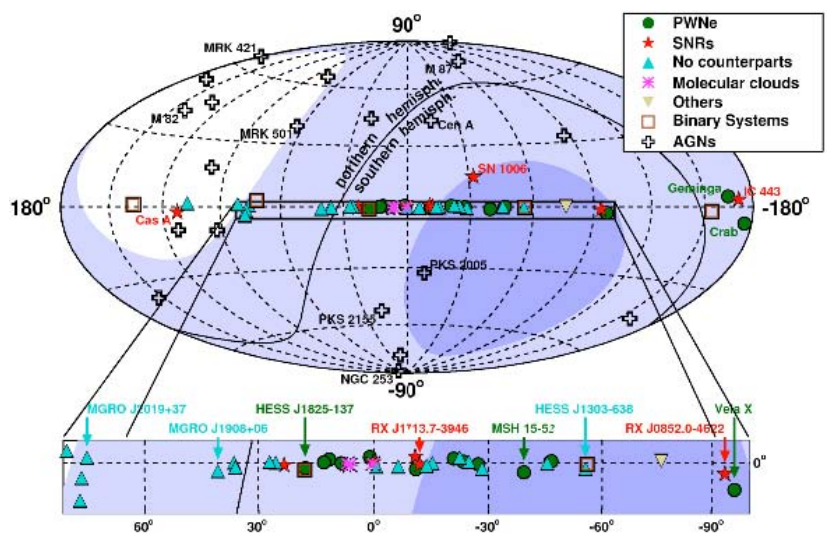

Fig. 1. Sky coverage in Galactic coordinates for detectors located in the Mediterranean Sea and at the South Pole, where only the Northern Hemisphere can be observed. The shading indicates the visibility for a detector in the Mediterranean with $2 \pi$ downward coverage; dark (light) blue areas are visible at least $75 \%(25 \%)$ of the time. The locations of observed sources of high-energy $\gamma$ rays are also indicated.

\section{General description}

The deep sea infrastructure KM3NeT consists of a neutrino telescope and a network of nodes for marine and Earth science investigations. The main goal of the neutrino telescope is the detection of high-energy (above some hundred $\mathrm{GeV}$ ) neutrinos of cosmic origin. The detection principle is based on the collection of Cherenkov photons emitted along the trajectory of charged particles that are produced in the interactions of neutrinos with rock and water in proximity of the telescope itself (Fig. 3).

Given the expected low neutrino flux at the source and the small neutrino cross section, a huge target mass is required together with an efficient shield against cosmic radiation. In the 1960s Markov (Markov, 1960) suggested the use of large volumes of natural water provided by the oceans, where seawater represents simultaneously the target, the shield and the active detection volume. A lattice of very sensitive optical detectors is deployed at a large depth under water (or ice). Time, position and charge information of signals due to Cherenkov photons are registered and processed with dedicated algorithms, allowing the reconstruction of the charge particle's direction. Though the telescope is sensitive to all neutrino flavors, it is optimized to neutrino-induced muons. Identifying upward going tracks allows the rejection of the overwhelming background of atmospheric muons. The angle between the neutrino and the muon directions is less than $1^{\circ}$ for neutrino energy larger than a few $100 \mathrm{GeV}$ (Fig. 4). An excess of tracks over the expected background of atmospheric neutrinos would provide evidence for a cosmic source. A complex analysis, based on sophisticated statistical tools, is required to estimate its statistical significance (Adrían-Martínez et al., 2011, 2012). The study of the energy spectrum of neutrinos

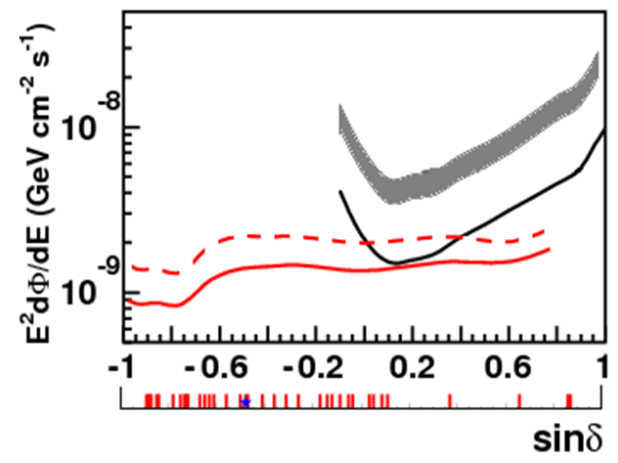

Fig. 2. Sensitivity of the KM3NeT detector to neutrino point sources with an $\mathrm{E}^{-2}$ spectrum for $1 \mathrm{yr}$ of observation, as a function of the source declination. The red lines indicate the flux sensitivity $(90 \%$ CL; full line) and the discovery flux $(5 \sigma, 50 \%$ probability; dashed line). The full black line is the IceCube sensitivity for $1 \mathrm{yr}$; the shaded band indicates the IceCube's discovery flux $(5 \sigma, 50 \%$ probability), spanning a factor 2.5 to 3.5 above the flux sensitivity. The red ticks show the positions of Galactic gamma ray sources, the blue star the position of the Galactic center.

reaching the detector can also provide indication of a diffuse flux of cosmic origin.

\section{Sites}

A suitable site to host this kind of infrastructure must fulfill several requirements, among which the following are the most relevant: sufficient depth in order to provide an effective shielding to the penetrating atmospheric radiation component; proximity to coast to facilitate deployment operations and to reduce the cable connection cost; high water transparency; low level of bioluminescence; low rate of biofouling and sedimentation on optical devices; low sea current velocity; and low risk of catastrophic events, like slope failures and significant earthquakes.

The KM3NeT project is based on the joined efforts of three European collaborations, ANTARES, NEMO and NESTOR, which, since a long time, have been performing accurate activities of site characterization. They have identified three different sites that have all been recognized as optimal sites for hosting such an infrastructure. Their locations are indicated in Fig. 5. For the time being, as available funds are strictly connected to regional considerations, the first steps of the KM3NeT construction will start in the Toulon and Capo Passero sites only.

\section{Technological solutions}

At the conclusion of the KM3NeT-DS project, a Technical Design Report (TDR) was published describing some different solutions that can be adopted for the realization of the neutrino telescope. A very short description of some key 


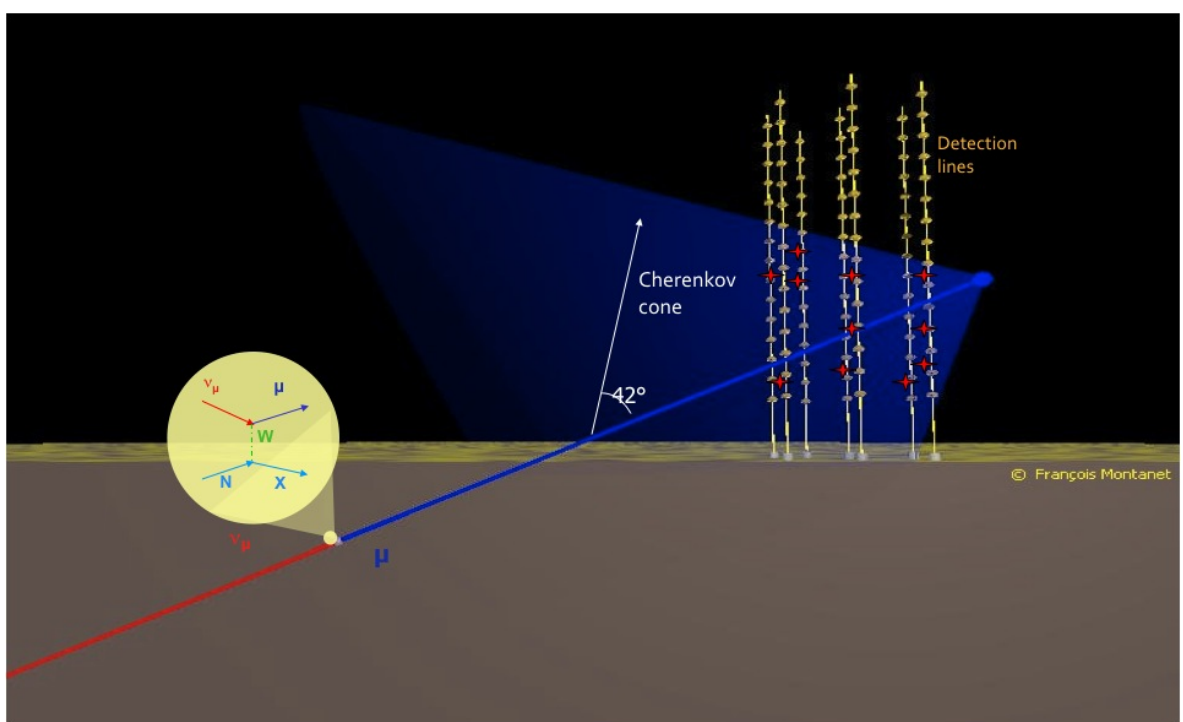

Fig. 3. Detection principle of neutrino-induced charged particles with an underwater telescope.

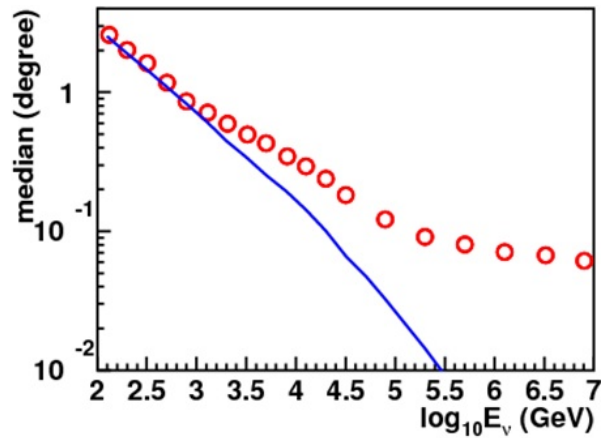

Fig. 4. Median of the distribution of the angle between the neutrino and the reconstructed muon (red points). The blue line is the median of the intrinsic angle between neutrino and muon directions, driven by the dynamics of deep-inelastic neutrino-nucleon scattering and kinematics.

components is given hereafter. For details the TDR is available on the KM3NeT site (http://www.km3net.org).

\subsection{Optical modules (OMs)}

The active part of a neutrino telescope is the optical module $(\mathrm{OM})$, which contains one or more photomultiplier(s) (PMT). It can be constructed following at least two different approaches:

- large PMT OM: it consists of a glass sphere hosting a large photomultiplier (PMT), $8^{\prime \prime}$ or $10^{\prime \prime}$ diameter. This solution meets the main requirements of a large photocathode area, a large angular coverage and a good timing response. It has the advantage of being a very welltested solution, used by the ANTARES (Amram et al.,
2002), the Baikal (Dzhilkibaev, 2004) and the IceCube (Abbasi et al., 2010) collaborations.

- multi-PMT OM: this is a prototypal design that foresees 31 PMTs of $3^{\prime \prime}$ diameter housed in a large glass sphere. The main advantages are a very large photocathode surface and the insensitivity to the Earth's magnetic field. In addition, the segmentation of the detection area in the $\mathrm{OM}$ can be useful in distinguishing single photon from multi-photon hits (Fig. 6) helping in the environmental background rejection.

\subsection{Detection units (DUs)}

Whichever design is chosen for the OMs, they will be attached to vertical structures anchored to the sea floor and kept almost vertical thanks to buoys at their top. Each structure is called detection unit (DU). Two main designs have been proposed for the mechanical structure of the DUs.

- Bar detection unit: this structure is a semi-rigid system composed of 20 horizontal elements (storeys), $6 \mathrm{~m}$ long, connected with a system of ropes forcing each storey to stay perpendicular to its vertical neighbors, $40 \mathrm{~m}$ apart. The number and the distance between storeys can be further optimized according to Monte Carlo simulations. The DU is anchored on the seabed and kept vertical by a buoy (Fig. 7). Each storey supports six optical modules (single or multi-PMT solution), the vessels hosting local storey electronics and some instrumentation for the positioning and the environmental monitoring systems (Fig. 8).

- String detection unit: this is a structure of 20 storeys, each comprising one multi-PMT OM, spaced vertically 


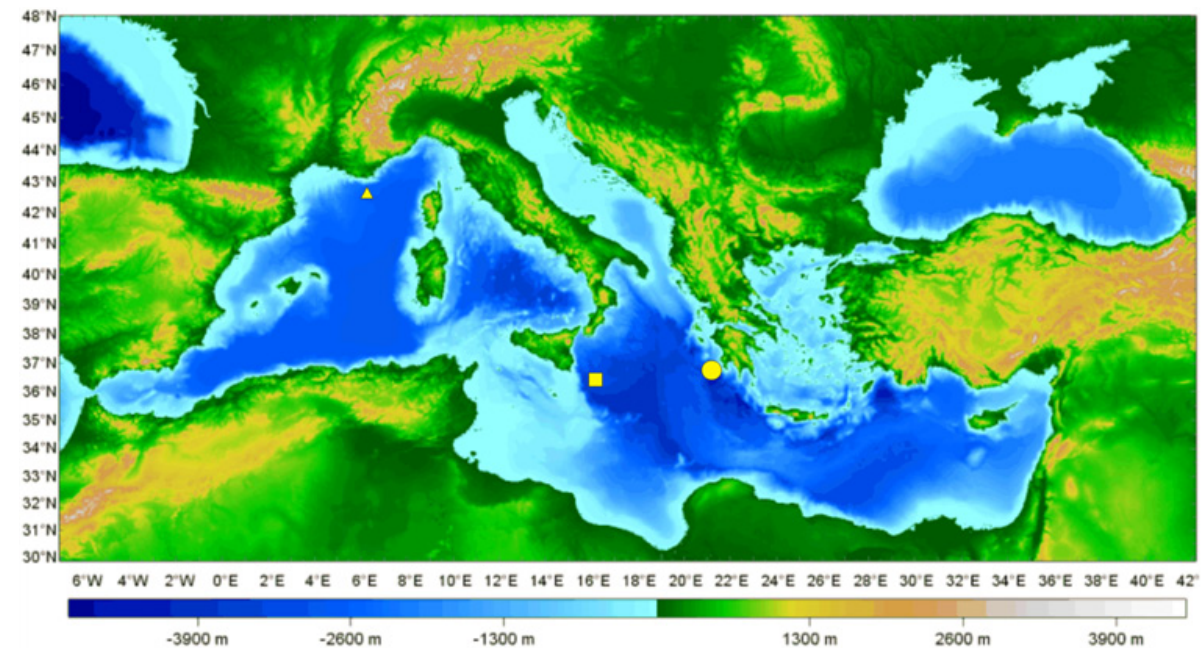

Fig. 5. Bathymetry of the Mediterranean Sea with the site locations marked. Triangle marks the Toulon site, ANTARES collaboration (Amram et al., 2003), the square the Capo Passero site, NEMO collaboration (NEMO coll., 2002) and the circle contains four possible sites near Pylos, NESTOR collaboration (Trimonis et al., 1992).

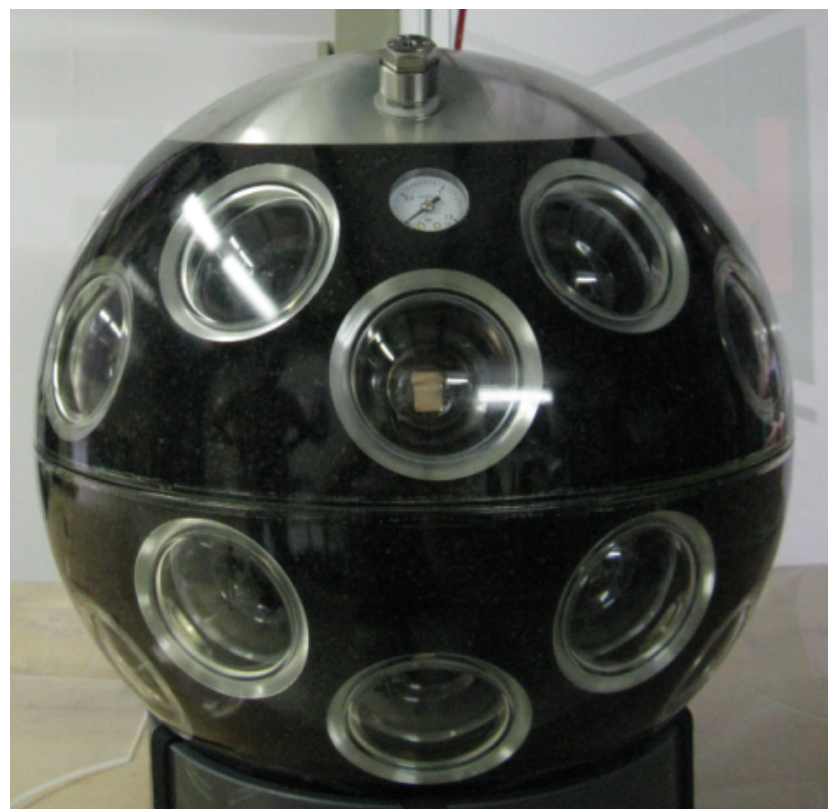

Fig. 6. Multi-PMT optical module, containing 31 PMTs with $3^{\prime \prime}$ diameter photocathode in a large glass sphere.

$30 \mathrm{~m}$ apart. The mechanical and electro-optical structures are separated, the former being provided by two ropes and the latter by a separate backbone cable. Also this DU is kept vertical thanks to a top buoy and anchored on the sea bed.

Successful deployment tests have been done with both structures.

MC simulations show that there is no significant difference in the performances of a neutrino telescope based on the more conventional technology of the large PMT OMs (associated with bar DUs) and what is expected from an advanced technological solution like the multi-PMT OMs with string DUs, the main advantage of the latter being a better rejection of the background due to bioluminescence and to atmospheric muons in case of an intermediate depth site. In particular performances are the same within $10 \%$, considering solutions whose costs differ by less than $20 \%$. Details on the design used in the simulations can be found in the KM3NeT TDR (http://www.km3net.org). A general agreement inside the consortium has been reached on the string DUs solution. Nevertheless, strict constraints in the timeline for the realization of the neutrino telescope in the Capo Passero site suggest starting the construction of a first part of the telescope following the design of the bar DUs with six large photocathode PMTs. When the innovative string DU with multi-PMTs are fully qualified, the integration with the traditional technology already present on the sea bottom will not represent an issue.

\subsection{DAQ and floor network}

A single cable from shore to a primary junction box provides the power required for the PMTs and the electronics. A network made with secondary junction boxes distributes power to the DUs and, via a vertical backbone cable, to the OMs. The same cable network hosts a fiber optic network for data transmission from PMTs to a shore station that houses computing facilities to filter and store the data from the telescope, before transferring them to remote nodes for analysis. The readout system converts the analogue output of the PMTs into formatted data for offline analysis. The expected total data rate is very high and can be transported on shore using optical fibers applying dense wavelength division 


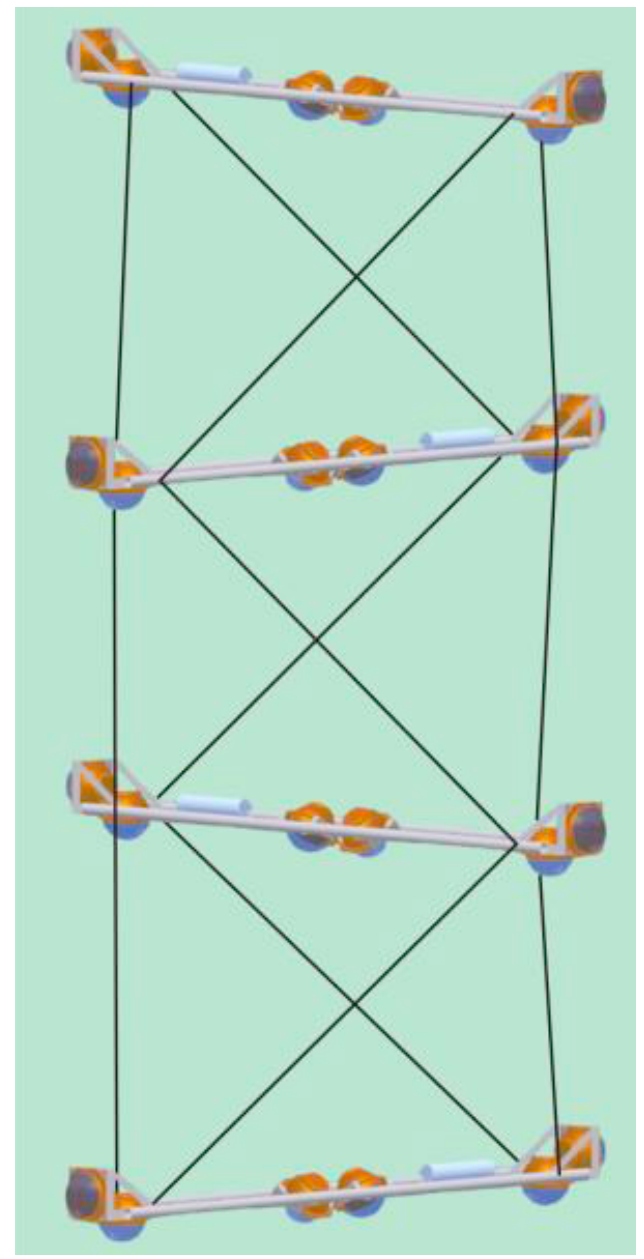

Fig. 7. A few storeys with interconnecting ropes for the bar detection unit (vertical scale is reduced).

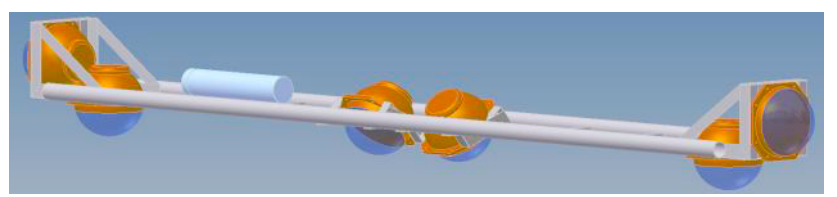

Fig. 8. The storey for the bar detection unit.

multiplexing (DWDM) techniques. Most of this flux is due to background signals, bioluminescence or ${ }^{40} \mathrm{~K}$ decay. At a first approximation, a detectable muon signal is defined by a number of causally correlated PMT signals above a predefined threshold. Some data filtering algorithms (triggers) are required to discriminate the neutrino/muon signal and to reduce the data storage requirements. Finally, a calibration system will determine the positions of the OMs and provide timing synchronization of the signals.
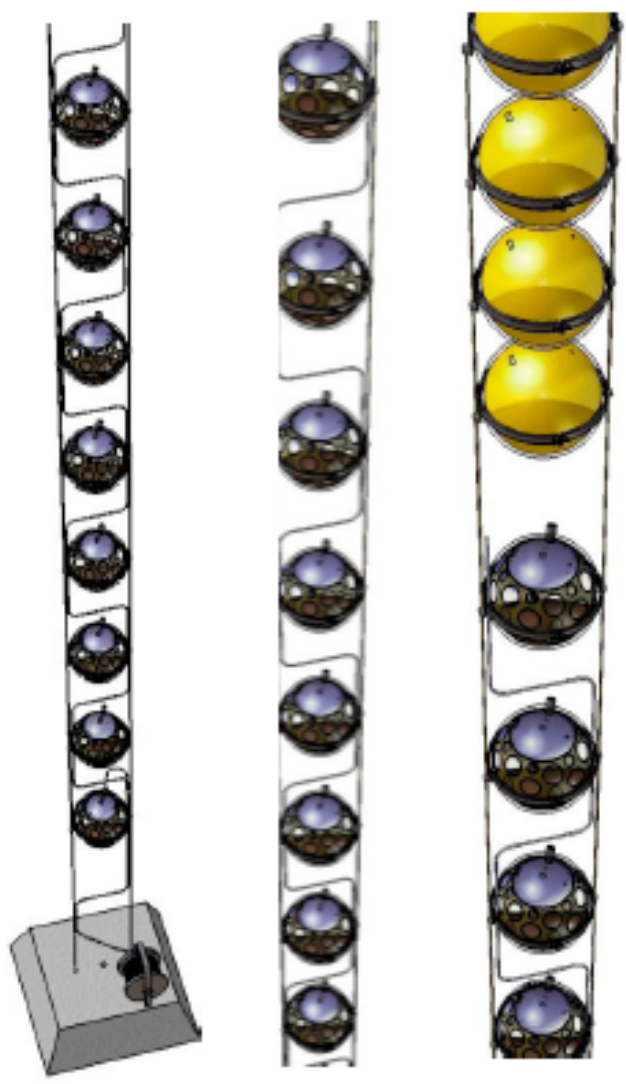

Fig. 9. Schematic layout of the string detection unit (from bottom at left to top at right).

\section{Earth and sea science}

The KM3NeT infrastructure will represent a unique opportunity for the Earth and sea science community to have a deep sea cabled observatory, enabling the collection of data at a high sampling rate, much larger than it is possible now and for a very long overall duration. Interactions between neutrino telescopes and sea science have already proved to be fruitful. Recent examples are van Haren et al. (2011) and Rubino et al. (2012). This will extend knowledge of some physical processes of the ocean and their interactions with biology and climate. Real time tracking of bioacoustic emissions will be possible. The system will also provide continuous observations to investigate the behavior of hazardous events such as earthquakes and might contribute to the regional tsunami early warning system under ICG/NEAM with better performance than data buoys currently deployed. The Earth and sea science component will practice a multidisciplinary approach and will form the basis of the Mediterranean section of the EU plan for long-term monitoring of the ocean margin environment around Europe and is part of the Global Monitoring for Environment and Security (GMES) system, complementing oceanographic networks such as GOOS (Global Ocean Observing System), 
EuroGOOS and DEOS (Dynamics of Earth and Ocean Systems). In addition, close cooperation will be pursued with EMSO (European Multidisciplinary Seafloor Observatory).

\section{Conclusions}

A short description of the KM3NeT project has been given. After years of study, a detailed design for large infrastructure including a $\mathrm{km}^{3}$ size neutrino telescope and a marine science observatory in the Mediterranean Sea has been prepared. In addition, the experience of the ANTARES telescope has demonstrated the feasibility of this technique. Some initial funds have been made available to start its construction in Capo Passero and Toulon sites. This is only the first step, but represents a fundamental step towards the realization of the whole project.

Acknowledgements. The research leading to these results has received funding from the European Community's Sixth Framework Programme under contract no. 011937 and the Seventh Framework Programme under grant agreement no. 212525.

Edited by: P. Strolin

\section{References}

Abbasi, R., et al. (IceCube Collaboration): Calibration and Characterization of the IceCube Photomultiplier Tube, Nucl. Instr. Methods, A618, 139-152, 2010.

Abbasi R., et al. (IceCube Collaboration): Time-integrated Searches for Point-like Sources of Neutrinos with the 40-string IceCube Detector, Astrop. J., 732, 18-33, 2011.

Abbasi, R., et al. (IceCube Collaboration): An Absence of Neutrinos Associated with Cosmic-Ray Acceleration in $\gamma$-Ray Bursts, Nature, 484, 351-354, 2012.
Adrián-Martínez, S., et al. (ANTARES Collaboration): First search for point sources of high energy cosmic neutrinos with the ANTARES neutrino telescope, Astrophys. J. Lett., 743, L14L19, 2011.

Adrián-Martínez, S., et al. (ANTARES Collaboration): Search for cosmic neutrino point sources with four years of data from the ANTARES telescope, Astrophys. J., 760, 53-62, 2012.

Amram, P. (ANTARES Collaboration): The ANTARES optical module, Nucl. Instr. Methods, A484, 369-383, 2002.

Amram, P. (ANTARES Collaboration): Sedimentation and Fouling of Optical Surfaces at the ANTARES Site, Astrop. Phys., 19, 253-267, 2003.

Dzhilkibaev, Zh.-A. (Baikal Collaboration): for the Baikal Collaboration: Comparison of $\mathrm{OM}$ responses at different sites, Proc. of the Very Large Volume Neutrino Telescope Workshop, 2003, 99-103, 2004.

Markov, M. A.: On high energy neutrino physics, Proc. 1960 Annual Int. Conf. on High Energy Physics, Rochester, p. 578, 1960.

NEMO coll.: http://nemoweb.lns.infn.it/upload/file/Siteselection/ Sitereport/NEMO-Site-Report.pdf (last access: 21 January 2013), 2002.

Rubino, A., Falcini, F., Zanchettin, D., Bouche, V., Salusti, E., Bensi, M., Riccobene, G., De Bonis, G., Masullo, R., Simeone, F., Piattelli, P., Sapienza, P., Russo, S., Platania, G., Sedita, M., Reina, P., Avolio, R., Randazzo, N., Hainbucher, D., and Capone, A.: Abyssal undular vortices in the Eastern Mediterranean basin, Nat. Comm., 3, 834, doi:10.1038/ncomms1836, 2102.

Trimonis, E. and Rudenko, M.: Geomorphology and bottom sediments of the Pilos area, in Proc. of 2nd NESTOR International Workshop, Pylos, 1992, available at: www.nestor.noa.gr/ 2nd/files/321_339_trimonis.pdf, 1992.

van Haren, H., et al. (ANTARES Collaboration): Acoustic and optical variations during rapid downward motion episodes in the deep north-western Mediterranean Sea, Deep-Sea Res. I, 58, 875-884, 2011. 